\title{
Rotatory quantization of charge-conjugation symmetric systems. 2. Harmonic and magneto-harmonic rotators
}

\author{
Zahid Zakir ${ }^{1}$
}

\begin{abstract}
For soft rotators the lack of a radial component of velocity is a defining property and it allows simplifying quantization of harmonic and magnetoharmonic rotators. Operators of observables of latter are normal ordered due to symmetries of the system, energy spectrum is linear on frequency and equidistant, and in the ground state there is no zero-point energy from rotational modes. It coincides with a generalization of the uncertainty relations for systems with nonhermitian canonical variables where the restrictions on fluctuations depend on state's charge. Applications of the new formalism to quantization of waves at collective rotations of one-dimensional chain of harmonic rotators allows to model fields with charge-conjugation and gauge symmetries. For the rotating modes there is a crossing symmetry between states with opposite rotation directions, and arising of negative-frequency modes are positive-frequency states of antiquanta with replaced initial and final states. The commutators and causal correlators (propagators) of generalized coordinates of the harmonic rotator are derived.
\end{abstract}

PACS: 03.65.Ge, 11.30.Er, 1130.Ly, 11.90. + t

Key words: discrete symmetries, rotations, charge-conjugation symmetry,

Landau levels, chain of rotators, propagators

\section{Content}

Introduction

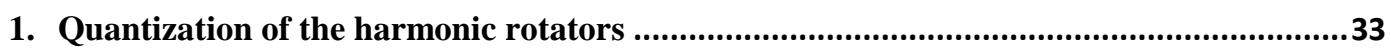

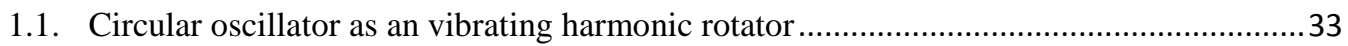

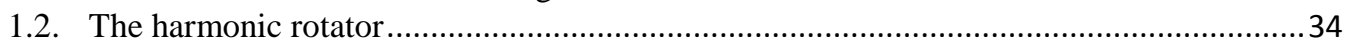

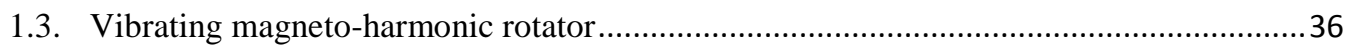

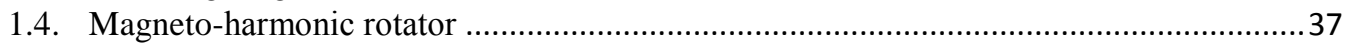

2. Discrete symmetries of rotators and their consequences .............................................39

2.1. The harmonic rotators with C-symmetry in terms of complex variables ........................39

2.2. Magneto-harmonic rotators with C-symmetry in terms of complex variables ...................40

3. The chains of rotators and the propagators .................................................................43

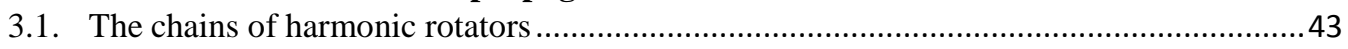

3.2. Quantization of a C-symmetric rotating chain of rotators ........................................ 44

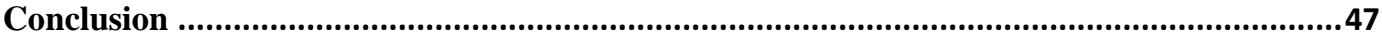

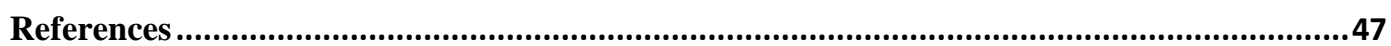

${ }^{1}$ Centre for Theoretical Physics and Astrophyics, Tashkent, Uzbekistan; zahidzakir@ theor-phys.org 


\section{Introduction}

Periodic processes in systems with harmonic potentials it is commonly accepted to reduce a set of harmonic oscillators, quantization vibrations of which gives an equidistant spectrum with a zero-point energy (vibrational quantization).

If periodicity in systems with harmonic potentials of purely rotational nature, here the physical meaning of observables changes and there appear new nontrivial properties. Particularly, in the model of a harmonic rotator - a particle in the harmonic potential on a plane without radial modes - frequencies are angular velocities of rotation and there the no zero-point energy from the rotational mode [1]. Further the approach where we quantize a purely rotational mode of the system, we will call as the rotatory quantization.

In the previous paper [2] the system of two charge-conjugate particles (a particle and its antiparticle) when each particle makes linear vibrations has been considered. The charge conjugation symmetry conditions have thus led to a spectrum without the zeropoint energy and this unusual fact also has appeared as related to rotational symmetry, but already on the complex plane of two degrees of freedom.

For clearing of physical reasons of the lack of the zero-point energy in the systems with purely rotational degrees of freedom in the present paper we will return to a one particle system with two degrees of freedom making pure rotations in the harmonic potential, the harmonic rotators, and their chains, and we will consider such models in more details.

Distinctions of rotational modes from the vibrational ones lead to essential distinctions of physical properties of the systems. At first, frequency means now angular velocity of rotations and consequently states on a rotation plane become double degenerate. Secondly, the angular momentum is conserved and naturally quantized even in the classical theory, expressing speed on which states differ at rotations on the same final angle for the same time. Thirdly, energy quantization turns out to be consequence of the angular momentum quantization and, fourthly, there is no zero-point energy from the purely rotational modes.

Therefore when the rotational modes in two-dimensional system are reduced to a set of the harmonic rotators the energy spectrum will be also equidistant and thus energy levels linearly depend on frequency and the angular momentum [1]. Generally the zeropoint energy from vibrational modes is present, but in some specific cases it can be compensated or does not arise at all. In particular, it is compensated at spin splitting of the ground state, instead of it does not arise at constraining the radial momentum or due to symmetries of system and in all these cases the energy spectrum starts with zero. Such cases without radial vibrations at all we will call as harmonic rotators, an example of which is a magneto-harmonic rotator.

In the paper the cases of harmonic rotators with exact and (spontaneously) broken charge conjugation symmetry are considered. It is shown that at exact charge conjugation symmetry the zero-point energy does not arise [2-3]. Models of the harmonic rotator with the zero-point energy of vibrational modes then correspond to the spontaneously-broken charge conjugation symmetry when the ground state is not symmetric.

In a chain of the harmonic rotators collective rotations around a common axis at small perturbation lead to transverse waves which at quantization give the quasi-particles and holes carrying an angular momentum. Group of symmetry thus becomes not $O(2)$, as for an isolated rotator, but the group $S U(2)$. It is related by the property of the chain of rotators when it transfers in itself not at rotation of on angle $2 \pi$, as an isolated rotator, 
but at its rotation on angle $4 \pi$, since it is possible to untangle the chain without a changing of boundaries (Dirac's method). As the result, in the chain naturally appear quanta in a spinor representation of the rotation group.

At inserting of interactions of quanta of the harmonic rotator on perturbation theory the operators observables in Hamiltonians and currents are automatically normal ordered. Therefore the normal ordering is in fact a part of the recipe of transition from the oscillatory to rotatory quantization.

In the first section of the paper quantization of the harmonic rotators of three types is presented. In section 2 quantization of charge conjugation symmetric rotators is considered and in section 3 the formalism is applied to quantization (transverse) waves of rotations in the chain of harmonic rotators. In section 4 the crossing symmetry of states are considered and correlators are found. Labels are same as in the paper [1].

\section{Quantization of the harmonic rotators}

\subsection{Circular oscillator as an vibrating harmonic rotator}

Quantization of motion of a particle in the harmonic potential on a plane it has been accepted to call as a circular oscillator problem though here are present both vibrational, and rotational modes. When the angular momentum is non-zero $M \neq 0$, the particle does not pass through centre because of prompt increasing of centrifugal energy and in fact the system behaves as a soft rotator. Therefore, further such system with $M \neq 0$ we will call as an vibrating harmonic rotator.

The standard solution of this problem is based on construction of the Hamiltonian and finding the wave equation solution. In the present paper we will begin with a Lagrange formulation of the problem and further we will single out contributions of rotational modes explicitly.

The Lagrangian of a vibrating harmonic rotator in polar coordinates on a plane of rotation looks like:

$$
\begin{aligned}
L & =\frac{1}{2} m\left(\dot{r}^{2}+r^{2} \dot{\theta}^{2}\right)-\frac{1}{2} m \omega^{2} r^{2}= \\
& =\frac{m}{2} \dot{r}^{2}+r^{2} \frac{m}{2}\left(\dot{\theta}^{2}-\omega^{2}\right) .
\end{aligned}
$$

The angle $\theta$ here is a cyclic variable and the angular momentum $M$ is conserved.

At the zero-eigenvalue of the moment $M=0$ there are pure vibrations along radius when the particle passes through centre and which it is possible to present as a superposition of two linear oscillators. But at nonzero angular moment $M \neq 0$, when the particle does not pass through centre, radii of levels are defined by equilibrium of centrifugal and elastic forces - the properties lacking for independent linear oscillators.

The Hamiltonian looks like

$$
H=\frac{p_{r}^{2}}{2 m}+\frac{M^{2}}{2 m r^{2}}+\frac{1}{2} m \omega^{2} r^{2}=\frac{p_{r}^{2}}{2 m}+U(r)
$$

and contains a kinetic energy of vibrations with a momentum $p_{r}$, and the centrifugal energy is included into an effective potential energy $U(r)$ :

$$
U(r)=\frac{M^{2}}{2 m r^{2}}+\frac{1}{2} m \omega^{2} r^{2} .
$$


Minima of total energy for various angular momenta correspond to lack of radial excitations and minima of $U(r)$. The latter are defined by $r_{e q}$, where centrifugal and elastic forces are balanced:

$$
U^{\prime}(r)=-\frac{M^{2}}{m r_{e q}^{3}}+m \omega^{2} r_{e q}=0, \quad r_{e q}^{2}=\frac{|M|}{m \omega} .
$$

Thus, the radial vibrations occur around these values of radius. Values of total energy at such equilibrium are equal to:

$$
E_{e q}=|M| \omega
$$

It is clear from (2) that at $M=0$ the system is reduced to two linear oscillators with the spectrum $E_{n_{r}, 0}=\hbar \omega\left(2 n_{r}+1\right)$. From this fact and from (5) it follows, that even at lack of radial excitations $n_{r}=0$, nevertheless there is the zero-point energy, and the contribution of rotational modes by taking into account of angular momentum quantization on a plane $M_{n}=n_{\theta} \hbar$, where $n_{\theta}=0, \pm 1, \ldots$, it is reduced to $\hbar \omega\left|n_{\theta}\right|$. Thus, for energy of rotational modes enlarged with adding of energy of zero-point radial vibrations we have

$$
E_{0, n_{\theta}}=\hbar \omega\left(\left|n_{\theta}\right|+1\right) \text {. }
$$

As a result, the energy spectrum of the vibrating harmonic rotator in general takes a form:

$$
E_{n}=\hbar \omega\left(\left|n_{\theta}\right|+2 n_{r}+1\right)=\hbar \omega\left(|Q|+2 n_{r}\right),
$$

where $|Q|=|M|+1=\left|n_{\theta}\right|+1$ is an effective "charge" of the state. The solution of the Schrödinger equation with (2) gives the same spectrum.

By introducing deviations from equilibrium radii $\rho=r-r_{n_{\theta}}$ and corresponding to them radial momenta of vibrations $p_{\rho}$, the Hamiltonian (2) can be written in the form:

$$
H=\frac{p_{\rho}^{2}}{2 m}+\frac{n_{\theta}^{2} \hbar^{2}}{2 m\left(r_{n_{\theta}}+\rho\right)^{2}}+\frac{1}{2} m \omega^{2}\left(r_{n_{\theta}}+\rho\right)^{2} .
$$

As we see, the diagram of energy - radius dependence looks like circular gutter with minima at purely rotational values of energy (6). It means that here we deal with a system with a "spontaneously broking" symmetry when vibrational levels are splitted on classes with a certain angular momentum quantum number $n_{\theta}$. Ground states of these classes are shifted on values of radius $r_{n_{\theta}}$, and minima of energies are lifted on values from (6).

This situation is opposite to a case of the molecular spectra where a difference of vibrational levels much more than rotational ones and the last start from certain vibrational level, forming "rotational bands" between two vibrational levels. In the vibrating harmonic rotator each rotational level makes a new ground state (gutter) from which the ladder of vibrational levels with the zero-point energy starts.

\subsection{The harmonic rotator}

In the case when a rotational energy sufficiently exceeds the radial kinetic energy, the properties of the vibrating harmonic rotators are determined mainly by the rotational modes. Therefore it is of interest to study them in a pure form by completely neglecting the radial kinetic energy. Such idealized systems with purely rotational kinetic term we will call as ideal harmonic rotators or simply harmonic rotators. 
The Lagrangian of the harmonic rotator contains a purely rotational kinetic term (as a rigid rotator) and an elastic potential (as a harmonic oscillator) [1]:

$$
L=\frac{1}{2} m r^{2} \dot{\theta}^{2}-\frac{1}{2} m \omega^{2} r^{2}=r^{2} \frac{m}{2}\left(\dot{\theta}^{2}-\omega^{2}\right) .
$$

Here $r^{2}$ enters in the Lagrangian uniformly and it can be taken out of the brackets as a Lagrange multiplier $\lambda=r^{2}$. This means that there $r$ is not a dynamic variable and for it the momentum does not introduced. The Lagrangian (9) is reduced to a pure constraint $L=\lambda \varphi(\dot{\theta})$ and the resolution of this constraint gives independence of angular speed (rotation frequency) $\dot{\theta}=\omega_{ \pm}= \pm \omega$ of radius. The cyclicity of the angular coordinate leads further to the angular momentum conservation $M_{ \pm}=m \omega_{ \pm} r^{2}=$ const that allows us to express the radii through the angular momenta: $r^{2}=M_{ \pm} / m \omega_{ \pm}$.

The Hamiltonian following from the Lagrangian (9) is:

$$
H=p_{\theta} \dot{\theta}-L=\frac{M^{2}}{2 m r^{2}}+\frac{1}{2} m \omega^{2} r^{2}
$$

On a surface of constraint $\varphi(\dot{\theta}) \approx 0$ it has a very simple form:

$$
H_{\dot{\theta}=\omega_{ \pm}}=M_{+} \omega_{+}+M_{-} \omega_{-} \text {. }
$$

Canonical quantization with introduction of a radial momentum $p_{r}$ and primary constraint for it $\varphi_{1}=p_{r} \approx 0$, leads to the same result, as the given simple derivation where the property of $r$ as a Lagrange multiplier has been used [1]. Then the angular momentum eigenvalues $M_{n}=n_{\theta} \hbar$ give an energy spectrum of the rotational levels:

$$
E_{n}=\hbar \omega\left|n_{\theta}\right| \text {. }
$$

The acceptance of the model Lagrangian (9) is an idealization supposing a possibility to neglect by the radial vibrations. This simplification is unfair for the systems containing the zero-point energy from the radial modes. Here it is important the fact that the rotational levels do not contribute to the zero-point energy. Therefore if in any realistic system with rotations the observations do not found out the zero-point energy it mean that in this system the purely rotational modes without an admixture of the radial vibrations are realized.

So, at comparison of harmonic rotator's energy spectrum with linear harmonic oscillator's spectrum there are three common properties and four distinctions. The common properties consist that 1) frequencies of all levels are identical, 2) the diagram of dependence of energy from radial extension is a parabola and 3) the energy levels are equidistant. New properties of the harmonic rotator are that its energy levels are 1) double degenerate, 2) proportional to the angular momentum $M, 3)$ do not contain the zero-point energy, and 4) are quantized because of the angular momentum quantization.

These properties of the harmonic rotator are characteristic for many systems with complex canonical variables, in particular, for relativistic fields and, consequently, their quantization it is natural to model on the basis of the this simple system. 


\subsection{Vibrating magneto-harmonic rotator}

An example of a realistic vibrating harmonic rotator represents a charged particle moving in a constant uniform magnetic field $H_{z}$. Motion in such field in the classical case occurs on circular orbits on a rotation plane $x, y$ with a cyclotron frequency $\omega_{H}=e H_{z} / m c \equiv 2 \omega_{ \pm}= \pm 2 \omega$ and radius $r_{H}=v_{\perp} / \omega_{H}$. Here the sign $\omega_{ \pm}$is determined by a sign of product $e H_{z}$ and consequently at a simultaneous changing of signs of the charge and the magnetic field (charge conjugation) frequency will not vary, which express a charge conjugation symmetry of the system.

The magnetic field acts, at first, as the elastic attractive force and, consequently, the system behaves as an vibrating harmonic rotator. Secondly, the rotation frequency on the circle twice more than frequency of a rotator with the same potential so the equilibrium radius is accordingly in $\sqrt{2}$ times less, than at the harmonic rotator with the same angular momentum. Thirdly, the field leads to the additional magnetic and spin splitting of levels. Taking into account all these similarities and distinctions further such system we will call as a vibrating magneto-harmonic rotator (see [1]).

Standard treatment of the magneto-harmonic rotator is based on the Hamiltonian formulation. To emphasize relationship with the vibrating harmonic rotator, we will present here a Lagrangian formulation also. The Lagrangian of the charged particle in the constant uniform magnetic field (while without spin)

$$
L=\frac{m}{2}\left(\dot{x}^{2}+\dot{y}^{2}+\dot{z}^{2}\right)+\frac{e}{c} \mathbf{A v},
$$

at choosing the vector potential in the form $\mathbf{A}=\mathbf{H} \times \mathbf{r} / 2$ has the form

$$
L=\left[\frac{m}{2}\left(\dot{x}^{2}+\dot{y}^{2}\right)+m \omega_{ \pm}(x \dot{y}-y \dot{x})\right]+\frac{m}{2} \dot{z}^{2}=L+\frac{m}{2} \dot{z}^{2} .
$$

Symmetries of the system become apparent in cylindrical coordinates, where a Lagrangian on the rotation plane $L_{\perp}$ in (14) takes a form:

$$
\begin{aligned}
L_{\perp} & =\frac{m}{2}\left[\dot{r}^{2}+\left(\dot{\theta}+\omega_{ \pm}\right)^{2} r^{2}\right]-\frac{m \omega^{2}}{2} r^{2}= \\
& =\frac{m}{2} \dot{r}^{2}+r^{2} \frac{m}{2}\left[\left(\dot{\theta}+\omega_{ \pm}\right)^{2}-\omega^{2}\right] .
\end{aligned}
$$

We see that this Lagrangian is similar to the Lagrangian of a vibrating harmonic rotator (1) and differ only by an angular speed shift $\dot{\theta} \rightarrow \dot{\theta}+\omega_{ \pm}$. The generalized momenta

$$
p_{r}=m \dot{r}, P_{\theta}=\frac{\partial L}{\partial \dot{\theta}}=m r^{2}\left(\dot{\theta}+\omega_{ \pm}\right)
$$

allow to express through them velocities:

and the Hamiltonian:

$$
\dot{r}=\frac{p_{r}}{m}, \dot{\theta}=\frac{P_{\theta}}{m r^{2}}-\omega_{ \pm}
$$

$$
H_{\perp}=p_{r} \dot{r}+P_{\theta} \dot{\theta}-L_{\perp}=\frac{p_{r}^{2}}{2 m}+\frac{P_{\theta}^{2}}{2 m r^{2}}+\frac{m \omega^{2}}{2} r^{2}-P_{\theta} \omega_{ \pm} .
$$


At quantization the radial vibrations formally contribute to energy $\hbar \omega\left(2 n_{r}+1\right)$. But, motions on the rotation plane are symmetric under both axes and in the classical case occur only on circular orbits. In the quantum case this means that it is necessary to take $n_{r}=0$. An energy of zero-point radial vibrations of a charge thus remains, but is transformed by a magnetic field to energy of "zero-point rotations" on arc-wise paths and the lowest level on a plane of rotation with $n_{\theta}=0$ is reduced to $E_{0}=\hbar \omega=\hbar \omega_{H} / 2$. Further, from (15) it follows for the rotational modes:

$$
\dot{\theta}=-2 \omega_{ \pm}, \quad P_{\theta}=-m r^{2} \omega_{ \pm} .
$$

As the result, by using that $P_{\theta}=-i \hbar \partial / \partial \theta \rightarrow n_{\theta} \hbar$, the energy levels on the rotation plane take a form:

$$
E_{n_{\theta} \pm}=\hbar \omega\left(2\left|n_{\theta}\right|+1\right)=\hbar \omega_{H}\left(\left|n_{\theta}\right|+1 / 2\right) .
$$

At the account of a spin of fermion $s$ the ground level is split on two sublevels:

$$
E_{0, s}=\hbar \omega_{H}\left(s+\frac{1}{2}\right)=\left\{\begin{array}{ll}
\hbar \omega_{H}, & s=1 / 2 \\
0, & s=-1 / 2
\end{array} .\right.
$$

As the result, for a particle with $s= \pm 1 / 2$ the lowest energy shifts to zero $E_{0,-1 / 2}=0$, and the next level $E_{0,-1 / 2}=\hbar \omega_{H}$ and all subsequent ones appear as double degenerate:

$$
E_{n_{\theta} \pm}=\hbar \omega_{H}\left(\left|n_{\theta}\right|+s+1 / 2\right)=\left\{\begin{array}{l}
\hbar \omega_{H}\left(\left|n_{\theta}\right|+1\right), s=1 / 2 \\
\hbar \omega_{H}\left|n_{\theta}\right|, \quad s=-1 / 2
\end{array} .\right.
$$

The radii of these orbits remain as former ones and identical, their energies shift only. Transitions between them occur at emitting or absorbing a photon with spin flip of a state.

Thus, splitting of levels because of interaction of the magnetic field with the magnetic momentum $M \omega_{H} / 2$, arisen due to motion on the circular orbit, for the given sign of a charge reduces number of states twice, by excluding the contribution of states with a backward rotation direction. Splitting of levels because of interaction of a magnetic field with particle's spin $-\mu(\mathbf{s H})=s \omega_{H}$ shifts to zero the lowest energy of electron with orientation of spin opposite to a field direction. As a result, in this particular case a unique situation when the total energy of the system is equal to the purely rotational energy is realized.

\subsection{Magneto-harmonic rotator}

Equations of motion for a charged particle in the uniform magnetic field $H_{z}$ in the classical limit vanish radial velocity on a rotation plane (a magneto-harmonic rotator). In an exact solution of a quantum problem an energy spectrum contains also the contribution of rotational levels only, shifted on the radial vibration's zero-point energy.

But, the zero-point energy from vibrational modes can be compensated by energy of spin splitting and then the energy spectrum starts with zero (see section 1.2). Such spectrum is similar to a spectrum of a harmonic rotator and for correctness of this analogy, we will consider a model of an ideal magneto-harmonic rotator or simply a magneto-harmonic rotator, in which the zero-point energy initially misses because of a complete elimination of radial modes. Still the problem on what systems it can be realized, at the present stage is not essential yet. 
In this connection it is obvious that simplicity and obviousness of procedure of quantization of the harmonic rotator should be characteristic for the given system also. There the radial velocity is excluded not by equations of motion, but explicitly by omitting of a radial kinetic energy in the Lagrangian when $r$ becomes Lagrange multiplier, or by inserting a constraint.

Let us begin with the first and simple derivation, when instead of the Lagrangian (15) we introduce the same, but without a radial kinetic term and where the kinetic energy on a rotation plane is fully reduced to the rotation energy:

$$
L_{\perp}=r^{2} \frac{m}{2}\left[\left(\dot{\theta}+\omega_{ \pm}\right)^{2}-\omega_{ \pm}^{2}\right]=r^{2} \frac{m}{2}\left(\dot{\theta}+2 \omega_{ \pm}\right) \dot{\theta} .
$$

In this Lagrangian $r^{2}$ enters uniformly and it is a Lagrange multiplier $\lambda=r^{2}$, so now $r$ there is no dynamical variable and for it the momentum does not introduced.

Here the Lagrangian (23) is a pure constraint $L_{\perp}=\lambda \varphi(\dot{\theta})$, where $\varphi=m\left(\dot{\theta}+2 \omega_{ \pm} \dot{\theta}\right) / 2$, and the resolution of this constraint $\dot{\theta}=-2 \omega_{ \pm}$gives independence of the angular speed (rotation frequency) on radius. From expression for a generalized momentum and angular momentum quantization then follow:

$$
\begin{aligned}
& P_{\theta}=m r_{n_{\theta}}^{2}\left(\dot{\theta}+\omega_{ \pm}\right)=-m r_{n_{\theta}}^{2} \omega_{ \pm}=n_{\theta} \hbar, \\
& r_{n_{\theta}}^{2}=-\frac{P_{\theta}}{m \omega_{ \pm}}=-\frac{n_{\theta} \hbar}{m \omega_{ \pm}}, \quad \frac{n_{\theta}}{\omega_{ \pm}} \leq 0 .
\end{aligned}
$$

The Hamiltonian following from the Lagrangian (23)

$$
\begin{aligned}
H_{\perp} & =P_{\theta}\left(\frac{P_{\theta}}{m r^{2}}-\omega_{ \pm}\right)-r^{2} \frac{m}{2}\left[\left(\frac{P_{\theta}}{m r^{2}}\right)^{2}-\omega_{ \pm}^{2}\right]= \\
& =\frac{P_{\theta}^{2}}{2 m r^{2}}+\frac{m \omega_{ \pm}^{2}}{2} r^{2}-P_{\theta} \omega_{ \pm}
\end{aligned}
$$

on the surface of constraint

$$
\varphi=\frac{P_{\theta}}{2 r^{2}}\left(\frac{P_{\theta}}{m r^{2}}+\omega_{ \pm}\right) \approx 0
$$

takes a very simple form:

$$
H_{\perp}=P_{\theta}\left(\frac{P_{\theta}}{m r^{2}}-\omega_{ \pm}\right)=-2 \omega_{ \pm} P_{\theta} .
$$

Corresponding excited levels of energy on the rotation plane:

$$
E_{n_{\theta}}=2 \hbar \omega\left|n_{\theta}\right|=\hbar \omega_{H}\left|n_{\theta}\right|
$$

also differ from (20) by lack of a radial zero-point energy $E_{0}=\hbar \omega_{H} / 2$ only.

Canonical quantization by introduction of a radial momentum $p_{r}$ and constraint $\varphi_{1}=p_{r} \approx 0$ yields the same result, as the given simple derivation using the property of $r$ as a Lagrange multiplier. Really, let us introduce the generalized Hamiltonian of a magneto-harmonic rotator $\tilde{H}_{\perp}$ which contains the Hamiltonian of a vibrating harmonic rotator and the primary constraint $\varphi_{1}=p_{r}$ :

$$
\tilde{H}_{\perp}=H_{\perp}+\lambda_{1} \varphi_{1},
$$




$$
H_{\perp}=\frac{p_{r}^{2}}{2 m}+\frac{P_{\theta}^{2}}{2 m r^{2}}+\frac{m}{2} \omega^{2} r^{2}-P_{\theta} \omega_{ \pm} .
$$

The constraint conservation condition is obeyed on the constraint surface:

$$
\left[\tilde{H}_{\perp}, \varphi_{1}\right]=\left[H_{\perp}, \varphi_{1}\right]=\frac{1}{2}\left[\frac{P_{\theta}^{2}}{m r^{2}}+m \omega^{2} r^{2}, p_{r}\right]=-\frac{P_{\theta}^{2}}{m r^{3}}+m \omega^{2} r \approx 0
$$

and another constraint does not arise. For more symmetrical form it is possible to introduce a constraint $\varphi_{1}=p_{r}^{2}$ and the result will be the same. On the surface of constraint the Hamiltonian $H_{\perp}$ from (30) transfers into (25) and gives the same spectrum (28), as at the simplified and the standard solutions of the problem.

\section{Discrete symmetries of rotators and their consequences}

\subsection{The harmonic rotators with $\mathrm{C}$-symmetry in terms of complex variables}

In classical rotating systems it is natural to introduce polar coordinates on the rotation plane with an angular variable $\theta(t)$. However, at quantization this variable leads to known paradox (see [3]): if to consider $\theta$ as an operator, in representation in which an angular momentum is diagonal, we obtain:

$$
\left(M-M^{\prime}\right)\left\langle M|\theta| M^{\prime}\right\rangle=-i \delta_{M M^{\prime}},
$$

that does not hold at $M=M^{\prime}$. This paradox is caused by that $\theta$ is not a uniquely defined variable and is not observable in a usual sense. But, the paradox disappears, if instead of $\theta$ to use a complex shift operator $\xi=\exp (i \theta)$. Then instead of (32) we obtain:

$$
\left(M-M^{\prime}\right)\left\langle M|\xi| M^{\prime}\right\rangle=\left\langle M|\xi| M^{\prime}\right\rangle,
$$

where it is visible that the matrix element is non-zero only for transitions $M-M^{\prime}=1$.

Transition from $\theta$ to $\xi=(x+i y) / r$ in fact is transition to a complex coordinate. In this connection we will consider a formulation of the harmonic rotators in terms of complex variables, which is not only more consecutive, but also it allows us to move forward and be closer to the problem of quantization of relativistic fields.

In a classical system with two real generalized coordinates $x, y$ and with the harmonic potential we will introduce the hermitian conjugate generalized coordinates:

$$
q=(x+i y) / \sqrt{2}, \quad q^{*}=(x-i y) / \sqrt{2}
$$

and the Lagrangian of such system looks like:

$$
L=m \partial_{t} q^{*} \cdot \partial_{t} q-m \omega^{2} q^{*} q
$$

This Lagrangian is invariant under the phase transformations

$$
q^{\prime}=e^{i \alpha Q} q, \quad q^{* *}=q^{*} e^{-i \alpha Q}
$$

with a constant parameter $\alpha$ and a conserved "charge" $\partial_{t} Q=0$, where

$$
Q=i\left(q^{*} p^{*}-p q\right) \text {. }
$$

The states of the system form two mutually "charge-conjugate" groups of states.

For the purely rotational modes of the system in the case of a harmonic rotator the radial momentum disappears. But since in the complex form the radial momentum explicitly is not singled out, for separation of the rotational modes from vibrational ones, some indirect approaches are required. 
Thus, the states are joined on two groups differing by a sign of the charge $Q= \pm 1$, transferring each other at the charge conjugation: $q_{c}=C q C^{-1}$. The Lagrangian, the Hamiltonian and the charge operator of such extended system look like:

$$
\begin{gathered}
L=\frac{1}{2}\left[m\left(\partial_{t} q^{*} \cdot \partial_{t} q-\omega^{2} q^{*} q\right)+m\left(\partial_{t} q_{c}^{*} \cdot \partial_{t} q_{c}-m \omega^{2} q_{c}^{*} q_{c}\right)\right], \\
H=\frac{1}{2}\left(\tilde{H}+\tilde{H}_{c}\right)=\frac{1}{2}\left[\frac{1}{m}\left(p p^{*}+m^{2} \omega^{2} q^{*} q\right)+\frac{1}{m}\left(p_{c} p_{c}^{*}+m^{2} \omega^{2} q_{c}^{*} q_{c}\right)\right], \\
Q=\frac{1}{2}\left(\tilde{Q}-\tilde{Q}_{c}\right)=\frac{1}{2}\left[i\left(q^{*} p^{*}-p q\right)-i\left(q_{c}^{*} p_{c}^{*}-p_{c} q_{c}\right)\right] .
\end{gathered}
$$

Since the system from two harmonic rotators does not contain the zero-point energy, such representation is formally identical to the considered in the previous paper [2] system of the harmonic potential with exact charge conjugation symmetry (of a particle and an antiparticle). Therefore further we cannot introduce explicit constraint into the Hamiltonian (39) for excluding a radial mode and to introduce only the exact charge conjugation symmetry requirement easily written in terms of complex variables.

As well as in [2], then the operators of observables are related to bilinear products $a^{*} a$ and $b^{*} b$ mutually charge-conjugate operators, giving zero at acting on the ground state (the "strong" normal ordering), by the relations:

$$
\begin{gathered}
a^{*} a=\frac{1}{2} i\left(q^{*} p^{*}-p q\right)+\frac{1}{2 m \omega}\left(p p^{*}+m^{2} \omega^{2} q^{*} q\right)=\frac{1}{2}\left(Q+\frac{1}{\omega} H\right)=\tilde{Q} . \\
b^{*} b=\frac{1}{2} i\left(q_{c}^{*} p_{c}^{*}-p_{c} q_{c}\right)+\frac{1}{2 m \omega}\left(p_{c} p_{c}^{*}+m^{2} \omega^{2} q_{c}^{*} q_{c}\right)=\frac{1}{2}\left(Q_{c}+\frac{1}{\omega} H_{c}\right)=\tilde{Q}_{c} .
\end{gathered}
$$

Althrough them we can express the charge operator and the Hamiltonian of states with a definite charge:

$$
\begin{gathered}
Q=\tilde{Q}+\tilde{Q}_{c}=a^{*} a-b^{*} b, \\
H=\omega\left(\tilde{Q}-\tilde{Q}_{c}\right)=\omega\left(a^{*} a+b^{*} b\right) .
\end{gathered}
$$

Here there is no the zero-point charge and zero-point energy that is of interest at application of this formalism to the relativistic fields.

\subsection{Magneto-harmonic rotators with $\mathrm{C}$-symmetry in terms of complex variables}

In the case of a magneto-harmonic rotator in complex variables on a rotation plane there are two kinds of Lagrangians, each of which transfers into the classical Lagrangian (14) and consequently intuitively were considered as equivalent ones:

$$
\begin{gathered}
L_{0}=m \dot{q}^{*} \dot{q}-m \omega \cdot i\left(q^{*} \dot{q}-\dot{q}^{*} q\right), \\
L_{\perp}=\frac{1}{2} m\left(\dot{q}^{*} \dot{q}+\dot{q} \dot{q}^{*}\right)-m \omega \cdot i\left(q^{*} \dot{q}-\dot{q}^{*} q\right) .
\end{gathered}
$$

But, it is well known that in quantum theory the velocities in this system do not commute and two Lagrangians lead to different models. The generalized momenta

$$
p=m \dot{q}^{*}-i m \omega q^{*}, \quad p^{*}=m \dot{q}+i m \omega q
$$

allow to express through them the velocities:

$$
\dot{q}=\frac{1}{m} p^{*}-i \omega q, \quad \dot{q}^{*}=\frac{1}{m} p+i \omega q^{*},
$$


and because of presence in (48) both momenta and coordinates, the commutator of velocities is equal to:

$$
\left[\dot{q}, \dot{q}^{*}\right]=\frac{2 \omega}{m} .
$$

By taking into account this property the Lagrangians (45) and (46) we can rewrite as:

$$
\begin{gathered}
L_{0}=m\left[\left(D_{t} q\right)^{*}\left(D_{t} q\right)-\omega^{2} q^{*} q\right] . \\
L_{\perp}=m\left[\left(D_{t} q\right)^{*}\left(D_{t} q\right)-\omega^{2} q^{*} q\right]-\omega,
\end{gathered}
$$

where a "covariant" time derivative is $D_{t}=\partial_{t}+i \omega$ and it allows rewriting momenta as:

$$
p^{*}=m D_{t} q, \quad p=m\left(D_{t} q\right)^{*} \text {. }
$$

Unlike the velocities (48), the "covariant" velocities $D_{t} q$ and $\left(D_{t} q\right)^{*}$, which are momenta up to a coefficient (mass), commutate.

The Lagrangians (50) - (51) lead to the Hamiltonians:

$$
\begin{aligned}
& H_{0}=\tilde{H}+\omega Q=\tilde{H}-\omega(M+1), \\
& H_{\perp}=\tilde{H}+\omega(Q+1)=\tilde{H}-\omega M,
\end{aligned}
$$

where $\tilde{H}$ is a Hamiltonian of a vibrating harmonic rotator:

$$
\tilde{H}=\frac{1}{m}\left(p p^{*}+m^{2} \omega^{2} q^{*} q\right)
$$

From (53) it follows that the radial zero-point energy in $\tilde{H}$ is compensated and since in a magnetic field there are no radial excitations, only purely rotational modes of the harmonic rotator contribute to the total energy:

$$
H_{0}=2 \omega\left(\left|n_{\theta}\right|-n_{\theta}\right)=\left\{\begin{array}{c}
\omega_{H}\left|n_{\theta}\right|, n_{\theta}<0, \\
0, \quad n_{\theta}>0 .
\end{array}\right.
$$

where $\omega_{H}=2 \omega$. Together with second, also purely rotational contribution $\omega Q$, they form the total Hamiltonian for the vibrating harmonic rotator. Thus, as in the classical case, the motion occurs only on circular orbits, but without zero-point energy.

Hamiltonians (53) - (54) can be written also in the form:

$$
\begin{gathered}
H_{0}=\frac{1}{m}\left(p+i m \omega q^{*}\right)\left(p^{*}-i m \omega q\right)=2 \omega a^{*} a=\omega_{H} Q, \\
H_{\perp}=\frac{1}{m}\left(p+i m \omega q^{*}\right)\left(p^{*}-i m \omega q\right)+\omega=2 \omega a^{*} a+\omega=\omega_{H}\left(Q+\frac{1}{2}\right),
\end{gathered}
$$

There the "minimal" Lagrangian $L_{0}$ leads to the "minimal" Hamiltonian $H_{0}$ without zero-point energy and consequently such system we name as an ideal magnetoharmonic rotator or simply a magneto-harmonic rotator. From the symmetrized Lagrangian $L_{\perp}$ follows the symmetrized Hamiltonian $H_{\perp}$ with the standard spectrum, containing the zero-point energy $E_{(0)}=\omega_{H} / 2$ from the radial zero-point fluctuations (transformed by the field into random motion on arc-wise paths).

At spin splitting of the ground level for a particle with $s= \pm 1 / 2$ the lowest energy $H_{\perp}$ also shifts to zero $E_{0,-1 / 2}=0$, and the following level $E_{0,-1 / 2}=2 \omega=\omega_{H}$ and 
all subsequent levels even at the same rotation direction also are double degenerate depending on spin's orientation, and the Hamiltonian looks like:

$$
H_{\perp}=\omega_{H}\left[a^{+} a+\left(\begin{array}{l}
1 \\
0
\end{array}\right)\right] .
$$

The states of the magneto-harmonic rotator with $Q$ are determined so that corresponds to rotations of a particle with the positive charge, frequency and energy. Let us perform a charge conjugation in the system when the charge of a rotating particle becomes negative, but the magnetic field changes a sign also. There the frequency and energy remain unchanged, i.e. the magneto-harmonic rotator is charge conjugation symmetric. This allows us to obtain the observables of states with operators $b^{+}, b$ by charge-conjugate the observables of states with operators $a^{+}, a$ and to write the observables of the systems in the charge conjugation symmetric form:

$$
\begin{gathered}
Q=\tilde{Q}+\tilde{Q}^{c}=a^{+} a-b^{+} b, \\
H_{0}=\omega_{H} Q=\omega_{H}\left(a^{+} a+b^{+} b\right) .
\end{gathered}
$$

From comparison with (57)-(58) then we come to the same relations between the charge operators as for the harmonic rotator.

From the physical point of view to two signs of a charge of a particle here correspond two rotation directions of magneto-harmonic rotators with the same energies. Such double degeneration of rotational levels generates a discrete symmetry with conserved quantity - a magnetic moment $\boldsymbol{\mu}_{z}$ proportional to a charge and an angular momentum of the rotating particle:

$$
\boldsymbol{\mu}_{z}=\frac{1}{2 m} e \cdot \mathbf{M}_{z} .
$$

If a sign of particle's charge changes $e \rightarrow-e$, the rotation direction in the magnetic field will change also $\omega_{+} \rightarrow \omega_{-}=-\omega$, it changes a sign of an angular momentum and, as the result, the magnetic moment remains unchanged: $\mu_{z} \rightarrow \mu_{z}$. The energy of interaction with the magnetic field becomes equal to:

$$
\boldsymbol{\mu}_{z} H_{z}=\frac{1}{2 m} e \cdot \mathbf{M}_{z} H_{z} .
$$

In the case of a simple harmonic rotator we will introduce a chiral charge $Q$ equal to a projection of an angular momentum of a quantum on a normal to a rotation plane $\mathbf{n}_{z}$ :

$$
Q_{n}=\mathbf{n}_{z} \cdot \mathbf{M}_{n} .
$$

The sign of the angular momentum is determined by a rotation frequency sign whereas the sign of $Q$ depends on a sign of orientation of $\mathbf{n}_{z}$ under the rotation plane. The chiral charge of the magneto-harmonic rotator is, of course, proportional to the ordinary charge of the rotating particle.

In the systems with charge conjugation symmetry the charge operator of the system figures instead of an angular momentum. A key symmetry of such systems is the symmetry between charge-conjugate states of the same sign of energy. As earlier it has been shown [2], at the Lagrangian in the "minimal" form this symmetry naturally 
excludes the zero-point energy and the zero-point charge. Therefore at quantization such systems are reduced not to a set of harmonic oscillators, but of harmonic rotators.

\section{The chain of rotators and the propagators}

\subsection{The chains of harmonic rotators}

The spectrum of a harmonic rotator equidistant, degenerate and does not contain the zero-point energy that takes place for quantized relativistic fields with the charge conjugation symmetries. Since the fields are similar to the continuous limit of chains of rotators, in [1] a model of a chain of rotators has been shortly considered. Here the formulation of the chain models in terms of complex variables will be presented.

Let there is a set of $N$ identical harmonic rotators, each of which is built as a system of two elastically connected particles $A_{i}$ and $B_{i}$, rotating on planes $\left(x, y, z_{i}\right)$ where $i=1, \ldots, N$, and let centers of mass of these rotators are located in line by step $d$ along a common rotation axis $z$. At identical angular speed $\omega_{0}$ and identical starting conditions the total set of rotators rotates coherently and, because of the angular momentum conservation, each of them remains on own rotation plane, and energy of the system is equal to:

$$
E_{N}=N_{+} M_{+} \omega_{0+}+N_{-} M_{-} \omega_{0-}+E_{0},
$$

where $M_{ \pm}$is an angular momentum of one a rotator.

Let us join by means of elastic force $\kappa\left(r_{i+1}-r_{i}\right)$ each of two particles of each of rotators to similar particles of two neighbor rotators ( $A_{i}$ with $A_{i \pm 1}$, and $B_{i}$ with $B_{i \pm 1}$ ), constructing a double one-dimensional chains of $2 N$ particles. In the continuous limit this double chain forms two massive strings joined by an elastic thin film. The rotation frequency of each of the harmonic rotators $\omega_{0}=\sqrt{\kappa / m}$ is constant and its connections with neighbors do not change it. They only lead to an angular momentum changing at an additional tension on a rotation plane, thus because of the angular momentum conservation and weakness of connections with neighbors, each rotator remains on own rotation plane.

Let now an external force adds to one of rotators in the chain an additional angular momentum and corresponding energy. This angular momentum will be transferred on the chain, while all chain will synchronously rotate as before, only small extra tension will move over the double chain. At a periodical perturbation a transverse wave of tensions of rotators will propagate in the chain. The length of this wave $\lambda=l^{\prime} d$ is determined by a number $l^{\prime}$ of the rotators covered by wave's period and corresponding wave vector $\mathbf{k}_{z}=2 \pi \mathbf{n}_{z} / \lambda$, directed along $z$, represents a momentum of wave's quanta. There with $\mathbf{k}_{z}$ it is related a "longitudinal" frequency of the propagating wave, defined as a product of $k_{z}$ on wave's velocity $\omega_{z}=v_{z} k_{z}$. Wave's crests of two opposite ends of rotators on a rotation plane $(x, y)$ rotate with the common rotation frequency $\omega_{0}$, move along $z$ with velocity $v_{z}$ and their trajectory in space form two helicoid curves around $z$ axis.

In quantum theory where each a rotator has a quantized angular momentum and corresponding energy levels, the wave's passing consists in transition to the next level 
and going back, so there is a propagation of a quasi-particle at adding of the angular momentum quantum and holes at its removing. If to the same rotator on the one hand chain the quasi-particle arrives, and on the other hand simultaneously a hole arrives, the angular momentum does not change and thus there is a recombination of these perturbations.

In a system of isolated rotators, also located along the same common rotation axis $\left(x_{i}, y_{i}\right)$ and coherently rotating, there is a rotational symmetry $O(2)$ in the space of two degrees of freedom with a conserved angular momentum. In a complex space $\left(q_{n}^{*}, q_{n}\right)$ a rotational symmetry corresponds to the group $U(1)$ with a conserved charge.

When rotators form the elastically bounded double chain, the connections of rotators with each other lead to a new effect of the topological nature - the system transfers into itself not at rotation one of rotators on angle $2 \pi$, as for the isolated rotator, but at its rotation on $4 \pi$, and thus a symmetry group at rotations becomes $S U(2)$. The reason is that, at rotation on $4 \pi$ one of the rotators, the chain can be untangled (by Dirac's method) and it is returned into a state before rotation without a changing of boundaries of the chain and without a changing of orientation of the turned rotator. Thus, in the chain of the bounded rotators there appear naturally quanta in the spinor representation of the rotation group.

\subsection{Quantization of a C-symmetric rotating chain of rotators}

The Lagrangian of a connected chain of harmonic rotators can be written as:

$$
L=\sum_{n=1}^{N}\left[m \dot{q}_{n}^{*} \dot{q}_{n}-k\left(q_{n+1}^{*}-q_{n}^{*}\right)\left(q_{n+1}-q_{n}\right)-m \omega_{0}^{2} q_{n}^{*} q_{n}\right]
$$

The canonical momenta

$$
p_{n}=m \dot{q}_{n}^{*}, \quad p_{n}^{*}=m \dot{q}_{n} .
$$

give the charge and the Hamiltonian:

$$
\begin{gathered}
Q=i \sum_{n=1}^{N}\left(q_{n}^{*} p_{n}^{*}-p_{n} q_{n}\right), \\
H=\sum_{n=1}^{N}\left[\frac{1}{m} p_{n}^{2} p_{n}^{* 2}+\kappa\left(q_{n+1}^{*}-q_{n}^{*}\right)\left(q_{n+1}-q_{n}\right)^{2}+m \omega_{0}^{2} q_{n}^{*} q_{n}\right] .
\end{gathered}
$$

The equations of motion following from this Hamiltonian are

$$
\begin{aligned}
& \ddot{q}_{n}+\omega_{0}^{2}\left(2 q_{n}-q_{n+1}-q_{n-1}\right)+\omega_{0}^{2} q_{n}=0, \\
& \ddot{q}_{n}^{*}+\omega_{0}^{2}\left(2 q_{n}^{*}-q_{n+1}^{*}-q_{n-1}^{*}\right)+\omega_{0}^{2} q_{n}^{*}=0 .
\end{aligned}
$$

In the continuous limit they transfer into the wave equations

$$
\begin{aligned}
& \frac{\partial^{2} q}{\partial t^{2}}-\mathrm{v}^{2} \frac{\partial^{2} q}{\partial r^{2}}+\omega_{0}^{2} q=0, \\
& \frac{\partial^{2} q^{*}}{\partial t^{2}}-\mathrm{v}^{2} \frac{\partial^{2} q^{*}}{\partial r^{2}}+\omega_{0}^{2} q^{*}=0 .
\end{aligned}
$$

with wave velocity $\mathrm{v}=\omega_{0} d$. These equations describe the transverse waves along two connected by an elastic film and rotating strings. rotators:

We can introduce periodic boundary conditions for a very large number of 


$$
q_{1}(t)=q_{N+1}(t), q_{1}^{*}(t)=q_{N+1}^{*}(t)
$$

Since there is a coherent rotation of all of rotators with common frequency $\omega_{0}$, the rotation energy of each of rotators is expresses through the angular momentum which now contains the contribution of frequency of the wave also. Thus, as before it is possible to present chain of rotators as a set of independent rotators (normal modes), but only with the frequencies $\omega_{k}$, following, as for a chain of linear oscillators, from the wave equation. Thus, it is possible to represent the waves on the chain of rotators as a series of independent normal modes of frequencies $\omega_{k}$, following from the wave equations (70):

$$
\ddot{a}_{k}(t)+\omega_{k}^{2} a_{k}(t)=0,
$$

where

$$
\begin{aligned}
& \omega_{k}^{2}=\omega_{0}^{2}\left(2-e^{i k d}-e^{-i k d}\right)+\omega_{0}^{2}=\mathrm{v}^{2} k^{2}+\omega_{0}^{2}, \\
& \mathrm{v}^{2} k^{2}=2 \omega_{0}^{2}(1-\cos k d) \approx k^{2} \omega_{0}^{2} d^{2} .
\end{aligned}
$$

All that allows us to decompose the generalized coordinates on frequencies of these normal modes:

$$
\begin{aligned}
& q_{n}(t)=\sum_{k} \frac{1}{\sqrt{2 m N \omega_{k}}}\left(a_{k} e^{-i \omega_{k} t+i k n d}+\beta_{k}^{*} e^{i \omega_{k} t-i k n d}\right), \\
& q_{n}^{*}(t)=\sum_{k} \frac{1}{\sqrt{2 m N \omega_{k}}}\left(a_{k}^{*} e^{i \omega_{k} t-i k n d}+\beta_{k} e^{-i \omega_{k} t+i k n d}\right),
\end{aligned}
$$

( $k=2 \pi l^{\prime} / N d, l^{\prime}-$ integer numbers). This decomposition corresponds to a case when at rotations of the doubled chain of the bounded rotators there is a propagation of transverse waves along $z$ axis with the wave numbers $k$ and frequencies $\omega_{k}$. Notice that the wavelength in the chain sufficiently exceeds distances between the rotators. The angular momentum, energy and the momentum of these waves are given by the expressions:

$$
\begin{aligned}
Q & =\sum_{k} Q_{k}=\sum_{k}\left(a_{k}^{*} a_{k}-\beta_{k} \beta_{k}^{*}\right), \\
H & =\sum_{k} \omega_{k} \cdot Q_{k}=\sum_{k} \omega_{k}\left(a_{k}^{*} a_{k}+\beta_{k} \beta_{k}^{*}\right), \\
P & =\sum_{k} k\left(a_{k}^{*} a_{k}+\beta_{k} \beta_{k}^{*}\right) .
\end{aligned}
$$

Commutators for the generalized coordinates and momenta give following nonzero commutators:

$$
\left[a_{k}, a_{k^{\prime}}^{*}\right]=\delta_{k k^{\prime}}, \quad\left[\beta_{k}, \beta_{k}^{*}\right]=\delta_{k k^{\prime}} .
$$

As the result, the charge operator and the Hamiltonian of normal modes take a form:

$$
\begin{aligned}
& Q_{k}=a_{k}^{*} a_{k}-\beta_{k} \beta_{k}^{*}, \\
& H_{k}=\omega_{k}\left(a_{k}^{*} a_{k}+\beta_{k} \beta_{k}^{*}\right) .
\end{aligned}
$$

Introducing corresponding $C$-conjugate ladder operators:

$$
\begin{aligned}
& b_{k} \equiv C a_{k} C^{-1}, \quad b_{k}^{*} \equiv C a_{k}^{*} C^{-1}, \\
& \alpha_{k} \equiv C \beta_{k} C^{-1},
\end{aligned}
$$

for the $C$-conjugate observables we obtain the expressions: 


$$
\begin{aligned}
Q^{c} & =\sum_{k} Q_{k}^{c}=\sum_{k}\left(b_{k}^{*} b_{k}-\alpha_{k} \alpha_{k}^{*}\right), \\
H^{c} & =\sum_{k} H_{k}^{c}=\sum_{k} \omega_{k}\left(b_{k}^{*} b_{k}+\alpha_{k} \alpha_{k}^{*}\right) .
\end{aligned}
$$

The difference of a wave on the chain of connected rotators from the common rotation by a constant frequency is in summation on normal modes with different wave frequencies. Since the modes with different frequencies are independent, each mode also is $C$-symmetric and the symmetry requirements relate the $C$-conjugate operators of observables in each mode:

$$
Q_{k}^{c}=-Q_{k}, H_{k}^{c}=H_{k},
$$

from which, by means of earlier shown procedure, follow the relations:

$$
Q_{-k}=-Q_{k}^{c}, \quad Q_{-k}^{c}=-Q_{k},
$$

or

$$
\alpha_{k} \alpha_{k}^{*}=a_{k}^{*} a_{k}, \quad \beta_{k} \beta_{k}^{*}=b_{k}^{*} b_{k} .
$$

By substituting them into (78) or (80), the observables can be expressed in terms of the operators of the mutually $C$-conjugate quanta:

$$
\begin{aligned}
Q & =\sum_{k} Q_{k}=\sum_{k}\left(a_{k}^{*} a_{k}-b_{k}^{*} b_{k}\right), \\
H & =\sum_{k} H_{k}=\sum_{k} \omega_{k}\left(a_{k}^{*} a_{k}+b_{k}^{*} b_{k}\right), \\
P & =\sum_{k} P_{k}=\sum_{k} k\left(a_{k}^{*} a_{k}+b_{k}^{*} b_{k}\right) .
\end{aligned}
$$

Thus, in the linear chain of harmonic rotators with $C$-symmetry a zero-point energy and a zero-point charge do not arise.

The commutator function of the generalized coordinates of the isolated harmonic rotator at direct evaluation is reduced to an odd solution of an equation of motion ( $f=1 / 2 m \omega)$ :

$$
\begin{aligned}
{\left[q\left(t^{\prime}\right), q^{+}(t)\right]=f } & \left(\left[a, a^{+}\right] e^{-i \omega\left(t^{\prime}-t\right)}-\left[\beta, \beta^{+}\right] e^{i \omega\left(t^{\prime}-t\right)}\right)= \\
& =f\left(e^{-i \omega\left(t^{\prime}-t\right)}-e^{i \omega\left(t^{\prime}-t\right)}\right)=-2 i f \sin \left[\omega\left(t^{\prime}-t\right)\right] .
\end{aligned}
$$

Further it is necessary to follow on causal restriction that quanta with positive energy evolve only forward in time, and quanta with the negative energy evolve only backward in time. Thus antiquantum evolution forward in time is described also as well as a usual quantum, but with the rearranged initial and final states. After elimination of negative-frequency states by crossing-transformation, there remain only quanta and antiquanta with positive energy which evolve only forward in time.

The commutator function for a chain of rotators looks like $\left(\Delta t=t^{\prime}-t\right)$ :

$$
\begin{aligned}
& {\left[q_{n^{\prime}}\left(t^{\prime}\right), q_{n}^{+}(t)\right]=\frac{1}{m} D\left[\Delta t,\left(n^{\prime}-n\right) d\right]} \\
& i D\left[\Delta t,\left(n^{\prime}-n\right) d\right]=\frac{1}{2 N} \sum_{k} \frac{1}{\omega_{k}}\left(e^{-i \omega_{k} \Delta t}-e^{i \omega_{k} \Delta t}\right) e^{i k\left(n-n^{\prime}\right) d} .
\end{aligned}
$$

The diagonal matrix elements from products of two generalized coordinates with different times $q^{+}(t) q\left(t^{\prime}\right)$ and $q\left(t^{\prime}\right) q^{+}(t)$ lead to the even causal correlator [2]. Therefore a causal propagator for the quanta proparating along the chain of rotators is: 


$$
\begin{aligned}
& i D_{c}\left[\Delta t,\left(n^{\prime}-n\right) d\right]= \\
& =m\left\langle 0\left|q_{n^{\prime}}\left(t^{\prime}\right)\right| 1\right\rangle\left\langle 1\left|q_{n}^{+}(t)\right| 0\right\rangle \theta(\Delta t)+\left\langle 0\left|q_{n^{\prime}}(t)\right| 1_{c}\right\rangle\left\langle 1_{c}\left|q_{n}^{*}\left(t^{\prime}\right)\right| 0\right\rangle \theta(-\Delta t), \\
& i D_{c}\left[\Delta t,\left(n^{\prime}-n\right) d\right]=\frac{1}{2 N} \sum_{k} \frac{1}{\omega_{k}}\left[\theta(\Delta t) e^{-i \omega_{k} \Delta t}+\theta(-\Delta t)\right] e^{i \omega_{k} \Delta t} e^{i k\left(n-n^{\prime}\right) d} .
\end{aligned}
$$

which is an even function of the time interval.

\section{Conclusion}

The analysis of applying in practice models of soft rotators allows to formulate their general theory and, in particular, quantum theory of a harmonic rotator. Operators of observables are normal-ordered due to the symmetries of system and there is no a zeropoint energy in the ground state. It is in the accordance with the generalization of uncertainty relations for such systems where restrictions on fluctuations depend on a value of a charge of the state.

Applications of the new formalism to quantization of waves at collective rotations of one-dimensional chain of harmonic rotators allow modeling the fields with gauge and $\mathrm{C}$-symmetries, and also with the spinor excitations. In the systems with rotational symmetries the crossing symmetry between states with opposite rotation directions takes place, and there arising negative-frequency modes represent positive-frequency antiquanta with rearranged initial and final states.

In the next paper the rotational quantization of fields, where a frequency of quantum means a rotation frequency of field vectors similar to a harmonic rotator, will be developed. There a new will be naturalness of inserting into the theory of charges and spin observables and also the lack of a zero-point energy and zero-point charge.

\section{References}

1. Zakir Z. Theor. Phys., Astrophys. and Cosmol., (2011) 6, 1, 1; doi: 6, 2, 14; doi:

2. Zakir Z. Theor. Phys., Astrophys. and Cosmol. (2006) 1,1,11; doi: 1, 4, 61; doi: ; (2007) 2, 2, 9; arXiv:0705.0899.

3. Paierls R. Surprises in Theoretical Physics., Princeton U. Pr, 1979. 